Verena Mayer, Mark Schlick und Martin Groeger

\title{
Landschaft als weicher Standortfaktor
}

\author{
Erarbeitung von Leitlinien, Zielen und projektbezogenen Handlungsvorschlägen \\ zur Sicherung und Entwicklung ästhetischer Landschaftsqualitäten
}

\section{Landscape as a Soft Locational Factor}

The development of guidelines, targets and project-based proposals for action to secure and develop aesthetic landscape qualities

\section{Kurzfassung}

Der vorliegende Beitrag beschäftigt sich mit der Erhaltung und Entwicklung landschaftlich ästhetischer Qualitäten des stark anthropogen geprägten Ballungsraumes Rhein-NeckarPfalz. Über eine Reihe von intensiven Analyseschritten werden innovative Konzepte entwikkelt, die dem Landschaftsraum ein Stück Identität zurückgeben und das Wohn- bzw. Arbeitsumfeld der dort ansässigen Bevölkerung aufwerten sollen. Der Landschaft kommt künftig als weichem Standortfaktor eine immer größere Bedeutung zu. Die Arbeit liefert Ansätze, wie im Rahmen eines Regionalen Landschaftsparks diesem Trend Rechnung getragen werden könnte und unterstreicht die Notwendigkeit baldigen Handelns, um die noch verbliebenen Freiräume des Ballungsraumes zu schützen und ästhetisch anspruchsvoll fortzuentwickeln.

\section{Abstract}

This article deals with the conservation and development of the aesthetic qualities of the largely man-made landscapes of the Rhine-Neckar-Palatinate conurbation. Proceeding through a series of stages of intensive analysis, the authors develop a number of innovative concepts for restoring to the landscape of this area some sense of identity as a means of enhancing the living and working environment experienced by the people who live there. Landscape quality is steadily gaining in importance as a soft locational factor. The present study puts forward a number of approaches suitable for giving this trend the recognition it warrants, for example within the framework of a Regional Landscape Park; it also underscores the need for immediate action in order to secure what open spaces still exist within this conurbation and to develop them in a manner which is aesthetically pleasing.

\section{Einleitung}

Der vorliegende Beitrag basiert auf den Ergebnissen eines Studienprojektes, das von den Verfassern im Zeitraum von Oktober 1998 bis Dezember 1999 an der Universität Kaiserslautern, Fachrichtung Raum- und Umweltplanung, durchgeführt wurde.

Ziel des Studienprojektes war es, beispielhaft für den Ballungsraum Rhein-Neckar-Pfalz gestalterische Leit- linien, Ziele und projektbezogene Handlungsvorschläge zur Sicherung und Entwicklung der ästhetischen Landschafts- und Freiraumqualitäten für unterschiedliche Planungsebenen zu erarbeiten und am Beispiel eines Projektes umzusetzen. Es sollte zugleich auf der Grundlage bisheriger methodischer Ansätze aufgezeigt werden, wie ästhetische Qualitäten einer Landschaft erfasst und gesteuert werden können. 
Für die Wahl des Themas waren folgende Gründe ausschlaggebend:

- Schöne Landschaften sind allgemein zu einem knappen Gut geworden. Gewachsene Kulturlandschaften drohen zu verfallen oder immer weiter überformt zu werden. Technische Elemente wie Verkehrstrassen, Freileitungen und Hochbauten prägen inzwischen vielerorts das Landschaftsbild. Ohne ästhetische Konzepte lassen sich diese Tendenzen nicht wirksam beeinflussen.

- Durch zahlreiche Untersuchungen ist der Nachweis erbracht worden, dass weiche Standortfaktoren (Stadt- und Landschaftsbild, Urbanität, Wohn- und Lebensqualität usw.) für die Entwicklung von Regionen und Städten immer wichtiger werden (u.a. Grabow u.a. 1995; Curdes 1989; Tochtermann u.a. 1995; Rothenburger u.a. 1997; Emnid 1995). Zu den Ursachen dieser Entwicklung gehören der sektorale und branchenstrukturelle Wandel mit einer zunehmenden Tertiärisierung, die wachsende Qualifikation der Beschäftigten, neue Techniken, eine Ausweitung der Arbeitsteilung, der Wandel der Zeitstrukturen oder das veränderte Verhältnis von Arbeit und Freizeit; Faktoren also, die in vieler Hinsicht Rückwirkungen auf die Standortanforderungen der Wirtschaft haben.

- Fragen der qualitativen Umweltgestaltung werden bei der Umsetzung des Leitbildes der Nachhaltigkeit (Agenda 21) immer bedeutsamer, da der umweltzerstörende Ressourcenverbrauch nicht allein durch Einsparung von Ressourcen und Effektivierung wirtschaftlicher Prozesse reduziert werden kann. Viel- mehr bedarf es einer Ergänzung durch eine Änderung des Lebensstils, und zwar eines Lebensstils, der nicht mehr das Glück in der Anhäufung materieller Güter verheißt. Um einen solchen Lebensstil zu fördern, ist es u. a. notwendig, auf eine Verbesserung der ästhetischen Qualitäten von Landschaft und Freiraum hinzuwirken, wobei sich diese Ansätze in ganzheitliche Konzepte einfügen müssen.

- Des Weiteren gewinnen Imagepflege und Standortwerbung im kommunalen und regionalen Bereich zunehmend an Bedeutung. Fischer stellt dazu treffend fest: „Wenn alle Städte und Regionen gleich gut aussehen, dann hilft nur der genius loci, die regionale kommunale Besonderheit weiter. Die jeweilige Identität wird man zwar herausarbeiten müssen, sie muss aber auch durch Imagepflege sowohl nach innen (Eigenimage), als auch nach außen (Fremdimage) transportiert werden. Wenn alle Städte und Regionen gleich gut funktionieren, wenn die "harten" Standortfaktoren (z.B. Infrastrukturangebot) überall ähnlich ausgeprägt sind, müssen die Unterscheidungsmerkmale in anderen Bereichen gesucht werden. Wenn die Bedeutung der "weichen“ Standortfaktoren, also beispielsweise die Lebensqualität, kurzum, der Imagewert des Raumes zugenommen hat, muss eben dies auch „kommuniziert" werden" (Fischer 1993).

Die Studienarbeit liefert einen Beitrag zur Bewältigung dieser aktuellen Herausforderungen räumlicher Entwicklung. Um möglichst weiterführende Anregungen für die Praxis zu liefern und Anstöße aus der Planungspraxis aufzunehmen, insbesondere im Kontext zum
Phase II

Historische Entwicklung

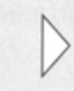

Phase II

Analysen/

Leitlinien

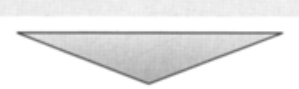

Teil 2

Ziele und Projektvorschläge für einen ausgewählten Teilraum M 1:20000

Phase VI Abgrenzung von Landschaftsbildeinheiten Phase V Erfassung der prägenden Merkmale Phase VI Erfassung der Gefährdungs- $\quad$ Projektvorschläge faktoren

Teil 3

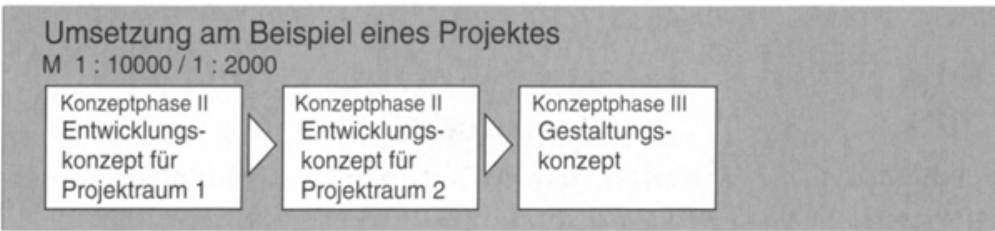

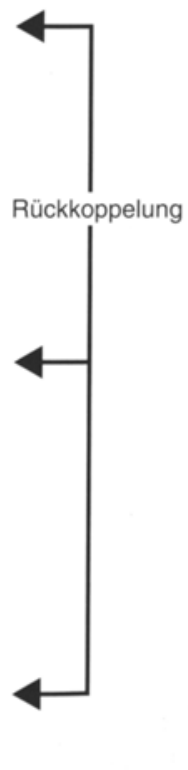


projektierten Regionalen Landschaftspark RheinNeckar-Pfalz, erfolgte eine frühzeitige Abstimmung mit dem Raumordnungsverband Rhein-Neckar. Die Abstimmung bezog sich sowohl auf die forschungsleitenden Fragestellungen als auch auf die inhaltiche Strukturierung der Untersuchung.

Der Arbeit lagen folgende Forschungsfragen zu Grunde:

- Welche Möglichkeiten und Grenzen bestehen bei der Erfassung von ästhetischen Qualitäten auf den unterschiedlichen Ebenen räumlicher Planung?

- Welche Zusammenhänge bestehen zwischen den einzelnen Planungsebenen?

- Welche Bedeutung kommt der historischen Landschaftsanalyse bei der Entwicklung von ästhetischen Konzepten zu?

In methodischer Hinsicht orientiert sich die Arbeit an dem von Schafranski (1996) entwickelten Analyseansatz zur Erfassung ästhetischer Qualitäten von Landschaften.

Die sich aus den Forschungsfragen ergebende inhaltliche Struktur der Arbeit ist in Abbildung 1 dargestellt.

Die einzelnen Arbeitsschritte werden nachfolgend begründet und erläutert und abschließend im letzten Kapitel kritisch reflektiert und in größere Zusammenhänge eingeordnet.

\section{2 Ästhetische Leitlinien für den Gesamtraum}

\subsection{Abgrenzung von Raumeinheiten}

Die Abgrenzung von Raumeinheiten bildet einen Grundschritt zur Erfassung der ästhetischen Qualitäten Vielfalt, Eigenart und Schönheit von Landschaften, wie sie im Zielparagraphen des Bundesnaturschutzgesetzes vorgeschrieben sind.

Anhand von Raumeinheiten lassen sich zum einen die landschaftlichen Eigenarten der Teilräume innerhalb eines Planungsraumes identifizieren und charakterisieren. Zum anderen ermöglichen sie Aussagen über die strukturelle Vielfalt der Landschaft.

Für die räumliche Strukturierung auf überörtlicher Ebene bietet sich die naturräumliche Gliederung Deutschlands an. Je nach Landschaftssituation sollten jedoch die naturräumlichen Einheiten durch landschaftsprägende Kriterien räumlich differenziert werden, um so verstärkt auf den spezifischen Charakter antworten zu können.

Bei der ästhetischen Analyse des Regionalen Landschaftsparks Rhein-Neckar-Pfalz erfolgten deshalb auf
Basis der bestehenden Siedlungsstruktur, der Struktur land- und forstwirtschaftlicher Flächen sowie der Gewässerstruktur weitere Untergliederungen, so dass auf diesem Wege 16 verschiedene Raumeinheiten abgegrenzt werden konnten, was auf eine hohe räumlichstrukturelle Vielfalt des Landschaftsparks hinweist.

Die planerisch-konzeptionelle Auseinandersetzung mit den Raumeinheiten hat gezeigt, dass die Methodik der Raumgliederung einen grundlegenden Einstieg in eine differenzierte Analyse der ästhetisch wirksamen Strukturen einer Landschaft darstellt. Es wäre jedoch problematisch, wollte man die vorgegebenen Raumstrukturen - die ein erstes Ordnungsmuster darstellen - unreflektiert in die Zukunft fortschreiben, da sie lediglich die dynamische Wechselbeziehung zwischen Natur und Mensch für einen bestimmten Zeitabschnitt wiederspiegeln.

\subsection{Historische Entwicklung der Landschaft}

Die Auseinandersetzung mit der natur- und kulturhistorischen Entwicklung einer Landschaft ist aus folgenden Gründen planungsrelevant:

- Sie erweitert das Wissen über eine Landschaft und ihre identitätsstiftenden Merkmale und schärft die Fähigkeit, ästhetische Qualitäten wahrzunehmen, und die Sensibilität gegenüber Eingriffen.

- Sie dokumentiert landschaftliche Veränderungen und Entwicklungstendenzen.

- Sie ermöglicht Konzepte für eine harmonische Landschaftsentwicklung.

- Sie stärkt den Bezug zur Landschaft und trägt zur Sinngebung und Wertebildung bei.

Über Umfang und Inhalte der historischen Analyse lassen sich keine generalisierenden Aussagen machen. Bis zu welchen Zeiträumen sie zurückreichen soll, muss von Fall zu Fall entschieden werden. Hiervon und von der Intensität der Analyse hängt es ab, ob Archivalien ausgewertet und Heimatforscher hinzugezogen werden müssen. Für eine plangraphische Kennzeichnung und Auswertung der landschaftlichen Entwicklung der letzten 100 bis 200 Jahre kann auf historisches Kartenmaterial unterschiedlicher Jahrgänge zurückgegriffen werden.

Die historische Analyse des Landschaftsparks erfolgte durch Gegenüberstellung von Topographischen Karten im Maßstab 1:100 000 aus den Jahren 1840/1930, 1965 und 1997. Untersucht wurde die Entwicklung der Gewässerstruktur, der land- und forstwirtschaftlichen Flächen, der Siedlungsflächen sowie der Technischen Infrastruktur. 
Bei der Analyse der geschichtlichen Entwicklung einer Landschaft darf nicht übersehen werden, dass der Blick zurück nicht zwingend zu Maßstäben führt. Diese müssen auf der Grundlage der heutigen Bedürfnisse entwickelt werden, damit zukunftsweisende und tragfähige Konzepte entstehen können.

\subsection{Erfassung ästhetischer Qualitäten und Gefährdungsfaktoren}

Aufbauend auf den abgegrenzten Raumeinheiten und den Ergebnissen der historischen Analyse lassen sich nun ausgewählte ästhetische Qualitäten sowie Gefährdungsfaktoren erfassen.

Bezüglich der ästhetischen Qualitäten können zwei Ebenen der analytischen Betrachtung unterschieden werden: zum einen die Analyse prägender Merkmale der Raumeinheiten und zum anderen die Untersuchung besonderer Voraussetzungen des Landschaftserlebens. Die prägenden Merkmale werden dabei durch einen Vergleich der Raumeinheiten anhand eines Kriterienkatalogs ermittelt, welcher auf die jeweilige Planungsebene auszurichten ist.

Bei den besonderen Voraussetzungen des Landschaftserlebens handelt es sich um solche Strukturen, die zwar in unterschiedlichen Landschaftsräumen vorkommen, aber auf Grund ihrer hohen Erlebniswirksamkeit besondere Erwähnung verdienen. Sie lassen sich durch die Auswertung von Karten und Bestandsaufnahmen vor Ort erfassen.

Beeinträchtigungen und Gefährdungen der ästhetischen Qualitäten können aus den Ergebnissen der historischen Analyse hergeleitet (Verlust und Veränderung von Merkmalen) sowie aus thematischen Karten (z. B. Lärmkarten) und durch Bestandsaufnahmen vor Ort festgestellt werden.

Von diesem methodischen Rahmen ausgehend, wurden ästhetische Qualitäten und Gefährdungsfaktoren erfasst. Zur gewählten Methodik bleibt kritisch anzumerken, dass sie zwar ein differenziertes Bild über vorhandene Qualitäten einer Landschaft vermitteln kann, vom Ansatz her aber nicht den Gesamteindruck wiedergeben kann, der beim Betrachter entsteht. Dieser Gesamteindruck ist bekanntlich mehr als die Summe der Teile. Soll ein Landschaftsraum differenziert gesteuert werden, führt an dem gewählten Ansatz kein Weg vorbei. Dem Nachteil dieser Methodik kann zumindest ansatzweise dadurch begegnet werden, dass an ausgewählten Standorten gewonnene Gesamteindrücke bei der Bewertung gezielt einbezogen werden.

\subsection{Entwicklung von Leitlinien}

Bei der Entwicklung ästhetischer Konzepte für die überörtliche Planungsebene ist darauf zu achten, dass die Vorgaben nicht zu eng gesetzt werden, um den schon bestehenden Tendenzen landschaftlicher Nivellierung und Verarmung nicht Vorschub zu leisten. Deshalb sollte sich das Konzept weitgehend auf Leitlinien beschränken, die Eckpunkte für die Sicherung und Entwicklung ästhetischer Qualitäten setzen.

Die gesamträumliche Analyse des Landschaftsparks hat gezeigt, dass die Region Rhein-Neckar-Pfalz stark anthropogen überformt ist und naturbelassene, vom Menschen weitestgehend unberührte Räume nahezu fehlen. Um eine weitere Beanspruchung der noch vorhandenen Freiraumpotenziale zu verhindern, müssen langfristig ausgerichtete, integrative Konzepte aufgestellt werden.

Zur Veranschaulichung werden nun einige Leitlinien wiedergegeben, welche in einem Plan räumlich umgesetzt wurden und auf die in nachfolgenden Bearbeitungsschritten in besonderer Weise reagiert wurde:

- Die Raumeinheiten sind - gemäß $§ 1$ Bundesnaturschutzgesetz - in ihrer Eigenart und Vielfalt zu erhalten, zu pflegen und zu entwickeln. Dazu gehört neben der Aufwertung und dem Schutz vorhandener Landschaftspotenziale auch ihre Vernetzung zu einem erlebbaren Gesamtsystem.

- Den Zersiedlungstendenzen, u.a. durch Entstehung von Siedlungsbändern, ist entgegenzuwirken.

- Durch Grün- und Siedlungszäsuren sind erlebbare Siedlungseinheiten und „intakte“ Freiräume sicherzustellen und die fortschreitende Zersiedlung der Landschaft zu vermeiden.

- Die Erhaltung optisch wirksamer Raumkanten und Dominanten ist im Landschaftspark langfristig anzustreben. Dazu gehört auch das Offenhalten von Aussichtspunkten und markanten historischen Sichtbeziehungen. Zwischen einzelnen Landschaftselementen sind zur Steigerung der Erlebbarkeit und der Orientierung Verbindungen zu schaffen.

- Die bestehenden Barrieren sind durch geeignete Maßnahmen zu überwinden, weitere Zerschneidungen sind zu vermeiden. In diesem Zusammenhang ist auch die Schaffung und Verbesserung der Querungsmöglichkeiten der Verkehrstrassen zu verwirklichen.

- Lokale Projekte sind als neue Identifikationsschwerpunkte und als „Trittsteine” innerhalb des Regionalen Landschaftsparks zu entwickeln.

- Durch den Aufbau eines großräumigen, vielseitigen Rad- und Fußwegenetzes ist die Erlebbarkeit der 
Landschaft zu steigern und der erforderlichen Vernetzung innerhalb des Landschaftsparks Rechnung zu tragen. Dabei sind historisch bedeutsame Wege und Achsen einzubeziehen.

- Gewerbeflächenausweisungen auf der „grünen Wiese" sind einzuschränken.

\section{Formulierung von Zielen}

\section{für einen ausgewählten Teilraum}

Die auf der Gesamtraumebene entwickelten Leitlinien stellen einen Ziel- und Handlungsrahmen zur landschaftlichen Entwicklung der nachfolgenden Stufe, den Teilräumen, dar. Dieser Rahmen muss durch räumlich konkretere und inhaltlich differenziertere Analysen und Bewertungen weiter ausgefüllt werden. Deshalb wird in der Studienarbeit beispielhaft der Raum Mannheim-Ladenburg-Heidelberg gewählt. Für dieses Gebiet wurde die historische Analyse durch Auswertung einschlägiger Veröffentlichungen sowie durch Befragungen von Zeitzeugen ergänzt.

\subsection{Abgrenzung von Landschaftsbildeinheiten}

Analog zum methodischen Vorgehen auf der Gesamtraumebene galt es im ersten Schritt, räumliche Bezugseinheiten zu bilden. Sie werden hier als Landschaftsbildeinheiten bezeichnet, weil sie bei Begehung in ihrer Unterschiedlichkeit und ihrem Charakter unmittelbar erlebt werden können. Die Abgrenzung stützte sich zum einen auf die Auswertung topographischer Karten, zum andern auf die Analyse von ausgewählten Standorten. Die Naturräumliche Gliederung eignete sich nicht mehr als Grundlage, da sie angesichts ihres kleineren Maßstabs $(1: 100000)$ die unterschiedlichen Landschaftstypen im Teilraum nicht zu erfassen vermag.
Im Untersuchungsraum lassen sich unter Einbeziehung der Flächennutzungen und der raumbildenden Strukturen drei deutlich voneinander abgrenzbare Landschaftsbildeinheiten ablesen:

1. Städtisch geprägte Einheit: „Verdichteter Siedlungsraum Mannheim“

2. Agrarisch geprägte Einheit: „Intensiv genutzte Landwirtschaftsflächen der Neckar-Rhein-Ebene"

3. Kulturhistorisch geprägte Einheit: „Gewachsene Kulturlandschaft Bergstraße"

\subsection{Erfassung der ästhetischen Qualitäten und der Gefährdungsfaktoren}

Die Erfassung ästhetischer Qualitäten und Gefährdungsfaktoren entspricht in methodischer Hinsicht dem erläuterten Ansatz auf der Gesamtraumebene. Die Unterschiede bestehen insbesondere in der inhaltlichen Differenzierung und der verstärkten Erfassung der Qualitäten vor Ort. Die für die Analyse des Teilraums herangezogenen Kriterien sind einer anderen Strukturierungsstufe der Landschaft zugeordnet als die Kriterien auf der Gesamtraumebene. Wurden beispielsweise auf gesamträumlicher Ebene Siedlungen in ihrem Gesamterscheinungsbild erfasst und bewertet, so spielen auf dieser Maßstabsebene die unmittelbar wahrnehmbaren Merkmalsausprägungen wie bauliche Strukturen und Nutzungen, die Stadtsilhouette, Ortseingänge und Ortsränder sowie Emissionen und Immissionen eine entscheidende Rolle. Die erfassten Qualitäten sowie die Gefährdungsfaktoren im Untersuchungsraum werden ebenfalls in Plänen dargestellt.

Durch diesen Analyseansatz werden ästhetische Potenziale und Defizite eines Landschaftsraumes verdeutlicht und eine Basis für die Entwicklung von Zielen und Projektvorschlägen geschaffen. Der festgestellte

Abbildung 2 Schematische Abgrenzung der verschiedenen Planungsebenen

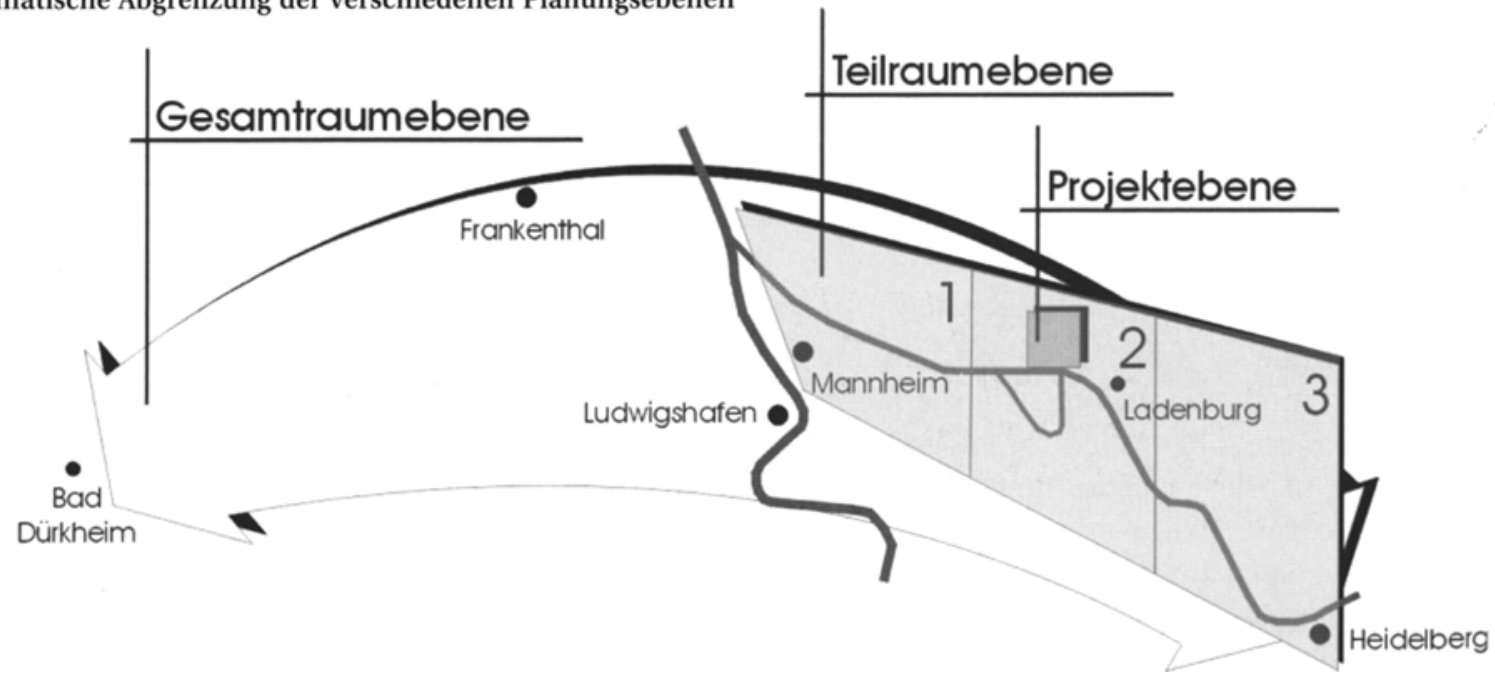


Mangel an qualitativ hochwertigen Freiräumen bzw. Naherholungsflächen sowie die durch starke anthropogene Überformung bewirkten strukturellen Nivellierungen und Verarmungen im Teilraum MannheimHeidelberg erfordern ein Ziel- und Handlungskonzept, das auch landschaftsästhetische Belange einschließt. Die nachfolgenden Ziel- und Handlungsvorschläge müssen sich umgekehrt in solche Konzepte einfügen und gegebenenfalls modifiziert werden.

\subsection{Zielvorschläge}

Die differenzierte Analyse auf der teilräumlichen Ebene erlaubt zwar einen enger formulierten Ziel- und Handlungsrahmen, gleichwohl muss darauf geachtet werden, dass sich dieser Rahmen nicht ins Negative verkehrt und statt zur Sicherung ästhetischer Qualität zu deren Verschlechterung auf lokaler Ebene beiträgt (z.B. durch Nivellierungseffekte und durch Vermeidung von räumlich differenzierenden Entwicklungen).

Folgende Ziele werden für die landschaftliche Entwicklung im Raum Mannheim - Ladenburg - Heidelberg zur Diskussion gestellt:

- Bei der zukünftigen Entwicklung des Teilraums sollten die charakteristischen Merkmale der drei Landschaftsbildeinheiten erhalten werden.

- Zur Verbesserung des Gesamteindrucks sind die freiraumästhetisch bedeutsamen Elemente zu einem Gesamtsystem zu verknüpfen und gegebenenfalls zu ergänzen. Visuell markante Landschaftselemente und Dominanten (z.B. Hangkanten, Talräume, Waldränder, Steinbrüche sowie Wassertürme) sind zu erhalten, da diese in entscheidendem Maße zur Erlebniswirksamkeit des Landschaftsraumes beitragen.

- Eine durchgängige Ost-West-Verknüpfung innerhalb des Teilraumes ist aufzubauen, um die strukturelle Vielfalt des Teilraumes und die Eigenarten der einzelnen Landschaftsbildeinheiten in räumlicher Abfolge erleben zu können.

- Durch die Ausweisung von Grünzäsuren ist zu verhindern, dass sich entlang der Bergstraße und des Neckars geschlossene Siedlungsbänder herausbilden.

- Die Attraktivität wohnungsnaher Erholungs- und Freiräume ist durch gestalterische und funktionale Aufwertung angrenzender ausgeräumter Feldfluren (z.B. mittels Raumbildung, Begrünung) zu verbessern. In diesem Zusammenhang sind neue bzw. attraktivere Wegeverbindungen aufzubauen, die nicht zuletzt zur Reduzierung und Überwindung der Zerschneidungswirkung vorhandener Infrastrukturtrassen beitragen können.
- Historische Bestandteile in der Landschaft (z.B. die Römerstraße, gewachsene Ortskerne, Altarme des Neckars, historische Sichtbeziehungen) sind als identitätsbildende Gestaltungselemente wiederaufzunehmen und zu integrieren.

Diese und weitere Ziele sind plangraphisch aufgearbeitet und verortet worden.

\section{Umsetzung der erarbeiteten Leitlinien und Ziele am Beispiel eines Projektes}

Wie aus Abbildung 3 hervorgeht, wird die Projektfindung und -konzipierung nicht allein durch Leitlinien und Ziele auf übergeordneten Planungsebenen bestimmt, sondern auch durch die situativen Verhältnisse und - insbesondere - durch die Kreativität des Planers.

Abbildung 3

Faktoren der Konzeptfindung

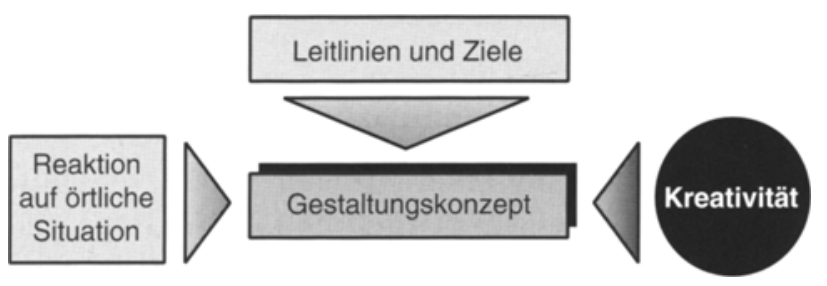

In der vorliegenden Studienarbeit wurden auf der Projektebene zunächst verschiedene Projekte entwickelt und im zweiten Schritt für das Projekt „Historische Achse: Römerstraße" ein Gestaltungsvorschlag erarbeitet.

Die Projektvorschläge und das Gestaltungskonzept umreißen ein Landschaftsbild, wie es in 10-20 Jahren aussehen könnte. Die Vorschläge sind beispielhaft durch verschiedene Entwicklungsszenarien visualisiert worden.

\subsection{Projektvorschläge}

\section{- Wege- und Wegeleitsysteme}

Bei der Gestaltung des Regionalen Landschaftsparks sind wiederkehrende Elemente einzusetzen, um den inneren Gesamtzusammenhang zu fördern und die Orientierung zu erleichtern. Dies wird durch Schaffung eines klaren Wege- und Wegeleitsystems innerhalb des Landschaftsparks unterstützt. Diese von Grünstreifen flankierten Wege dienen nicht nur als Verbindungslinien, sondern auch als richtungsweisende, gliedernde Raumelemente, die zudem optische und ökologische Bruchstellen überwinden. Um dem Besucher des 


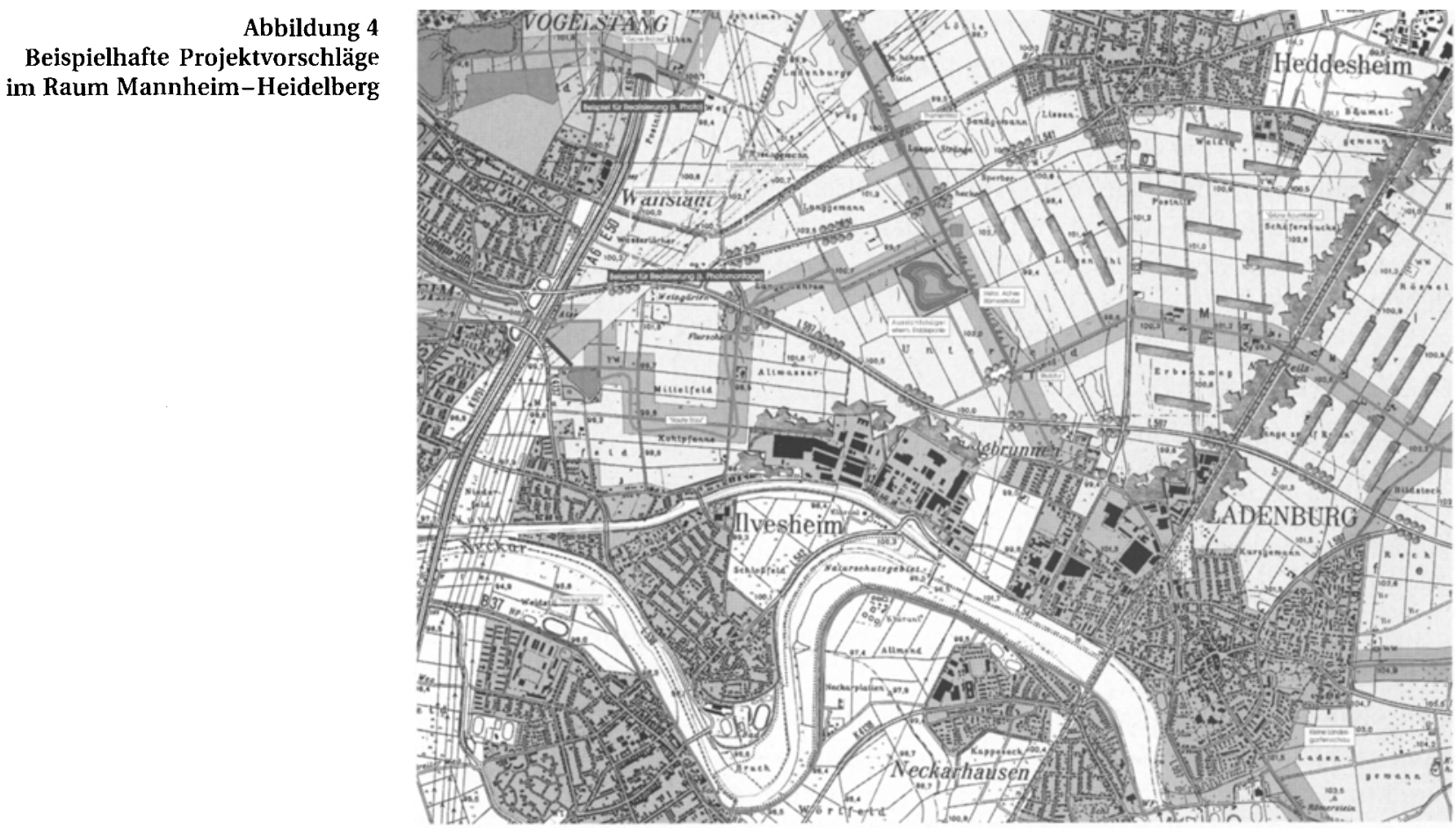

Landschaftsparks die besondere landschaftliche Situation sowie die Regionalparkidee zu vermitteln, werden an zentralen Orten Informationstafeln (z.B. über naturund kulturhistorische Besonderheiten) angebracht, die auf besondere Attraktionen des Parks hinweisen und gleichzeitig der Orientierung dienen. Kleine Informationscenter mit Bistro und Fahrradverleih können das Serviceangebot abrunden, um den Bedürfnissen Erholungssuchender zu entsprechen.

\section{- „Grüne Finger“}

Um die stetig steigende innerstädtische Flächeninanspruchnahme einzugrenzen bzw. zu koordinieren und die verbleibenden, in die Stadt ragenden Freiräume langfristig $\mathrm{zu}$ sichern, bedarf es klarer Nutzungskonzeptionen. Die Aufwertung und der Erhalt potenzieller bzw. bereits vorhandener Grünverbindungen aus der freien Landschaft in die Stadt und die Rückgewinnung, Verbesserung und Entwicklung von wohnungsnahen und qualitativen Erholungs- und Freiräumen für die Stadtbevölkerung (Bildung sog, ,grüner Nachbarschaften") steht hierbei im Vordergrund.

\section{- „Grüne Brücke“}

Gerade im Übergangsbereich der Siedlungen zur „offenen" Landschaft ist deren Zugänglichkeit meist auf Grund der Barrierewirkung von Verkehrstrassen nicht gewährleistet bzw. empfindlich beeinträchtigt. Aus diesem Grund ist die Herstellung großzügig dimensionierter, begrünter Autobahnüberquerungen (qualitativ hochwertige Überdeckelung bzw. Untertunnelung von Teilabschnitten der Autobahn-, Bundesstraßen- und
Schienentrassen, z.B.: „Grüne Brücken”) vorgesehen, welche eine qualitative Vernetzung der Landschafts räume ermöglichen.

\section{- Historische Achse: Römerstraße}

Diese Route im Regionalen Landschaftspark nimmt die historische Wegebeziehung der ehemaligen Römerstraße auf und thematisiert ihre Geschichte in verschiedenen Feldern am Wegerand. Auf diese Weise ent steht ein gestaltetes „Highlight“ in einer weitestgehend ausgeräumten und ungegliederten Ackerlandschaft.

\section{- „Grüne Raumteiler“}

Die „Grünen Raumteiler" stellen in der ausgeräumten Agrarlandschaft streng geometrisch angeordnete, an Feldflurgrenzen orientierte Gehölzriegel zur Gliederung des Landschaftsraumes. Neben ihrer ökologischen Wertigkeit und der Absicht, unterschiedliche Raumsequenzen zu bilden, können diese „Raumteiler unterschiedlicher Höhe auch besondere Blicksituationen betonen. In Zusammenhang mit diesem Projektvorschlag steht die Sanierung und Neupflanzung von Alleen.

\section{- „Route Blau“}

In Bezugnahme auf die historische Flusslandschaft des Neckars wird ein vom Wasser begleiteter Weg angelegt. In Form eines mäandrierenden Bachlaufes sowie verschiedener Wasserrinnen wird der ehemalige Flussverlauf aufgenommen. Bei diesem Projektvorschlag steht demnach die Erlebbarkeit des Elementes Wasser im Vordergrund. 


\section{- „Weg der Weitsicht“}

Die entlang der Bergstraße führende Route des Regionalen Landschaftsparks wird als "Weg der Weitsicht" bezeichnet, da sie auf Grund ihrer leicht erhöhten Lage (am Rande des Höhenzuges des Odenwaldes) den Blick bis zum Haardtrand (und damit über den gesamten Projektraum des Regionalen Landschaftsparks) ermöglicht.

\section{- „Weg der Umsicht“}

Die östlich von Ladenburg um die Stadt führende Route lenkt in einem bogenförmigen Verlauf die Blicke der Erholungssuchenden auf die Umgebung und somit auf die unterschiedlichen Landschaftsbildeinheiten des Teilraumes.

\section{- „Landart"-Projekte}

Eine dauerhafte Lichtinstallation am östlichen Stadtrand von Mannheim erfolgt als Initialzündung für weitere Kunstprojekte in der Landschaft und nimmt Bezug auf die den Landschaftsraum prägenden Hochspannungstrassen. Beispielhaft wird dabei ein Teilstück der Freileitungen unterirdisch verlegt und durch eine Laser-Illumination neu interpretiert. Ein Hochspannungsmast erhält als "Aussichtsgerüst" eine neue Funktion.

\section{- Integration „Kleine Landesgartenschau Ladenburg“}

Die "Kleine Landesgartenschau Ladenburg" ist in das Konzept des Regionalen Landschaftspark einzubinden, indem eine "modellhafte“ Verzahnung des Siedlungsrandes mit der offenen Landschaft gezeigt wird. Der besondere Stellenwert der Stadt Ladenburg liegt in ihrer zentralen Lage im Teilraum Mannheim-Heidelberg und der historischen Altstadt begründet. Sie stellt somit einen Dreh- und Angelpunkt für Erholungsuchende dar.

\section{- Langfristige Maßnahmen}

Als langfristiger und visionärer Ansatz für die Funktionsfähigkeit des Parks wird die unterirdische Verlegung der visuell störenden Hochspannungsleitungen vorgeschlagen, wodurch sich die ästhetischen Qualitäten steigern lassen und sich ein weiteres Spektrum an Gestaltungsmöglichkeiten für den Freiraum ergibt.

Aus finanziellen und technischen Gründen lassen sich einige Projektvorschläge wohl in absehbarer Zeit noch nicht realisieren (z.B. die Verkabelung der Hochspannungsleitungen). Die Einbeziehung heute noch visionär erscheinender Projekte ist in Anbetracht des langfristig ausgelegten Instrumentes „Regionaler
Landschaftspark“ aber nicht nur sinnvoll, die Erfahrung zeigt, dass oftmals in der Vergangenheit als unrealisierbar bezeichnete Ideen heute bereits selbstverständlich sind.

\subsection{Gestaltungskonzept für das Projekt „Historische Achse: Römerstraße“}

Die Römerstraße ist ein außerordentlich wichtiges Element innerhalb des Regionalen Landschaftsparks und daher in besonderer Weise für eine gestalterisch - konzeptionelle Konkretisierung geeignet. Die Entwurfsidee orientiert sich weniger an einer reinen Reflexion als an einer zeitgemäßen Neuinterpretation der Geschichte des Landschaftsraums. Die Kombination kulturhistorischer Landschaftselemente mit moderner Freiraumarchitektur wird daher im Gestaltungsvorschlag konsequent umgesetzt.

Vorrangiges Ziel des Gestaltungskonzeptes ist es, den anthropogen überformten Landschaftsraum aus landschaftsästhetischer Sicht aufzuwerten und die monoton wirkende Agrarlandschaft unter Berücksichtigung ihrer Eigenart zu bereichern.

Die Basis des Entwurfs bildet das städtebauliche Grundraster antiker römischer Castren. Die von Nord nach Süd verlaufende historische Römerstraße entspricht der ebenso ausgerichteten antiken Hauptachse "Cardo", welche im rechten Winkel von einem als "Decumanus" betitelten Wirtschaftsweg gequert wird. Entlang der beiden Hauptachsen erschließen sich in gleichen Abständen so genannte „insulae“ von $100 \times 100$ m. Diese neuen, ein Hektar großen Felder sind mit unterschiedlichen Freiraumfunktionen belegt, deren Nutzungsmöglichkeiten zwischen Spiel- bzw. Liegewiese bis hin zu stark architektonisch geprägten Inseln differieren. Dadurch wird außerdem eine Steigerung der Gestaltungsintensität von außen nach innen erzielt.

Das bewusst großzügig entworfene Wasserbecken inmitten der Anlage symbolisiert das „Forum" der römischen Städte ("Gewässer-Forum“). Die Strenge des Systems wird von einem natürlich gestalteten Bachlauf durchbrochen, der an den historischen Verlauf des Neckars erinnert (vgl. Projekt: „Route Blau“).

Die vier an den Eckpunkten des "Castrums" vorhandenen Aussichtsplattformen ermöglichen einerseits einen weiten Ausblick in das Umland und ein großräumiges Landschaftserleben. Andererseits machen sie den Erholungssuchenden als exponierte "Landmarken" auf die Freiraumkomposition aufmerksam. 


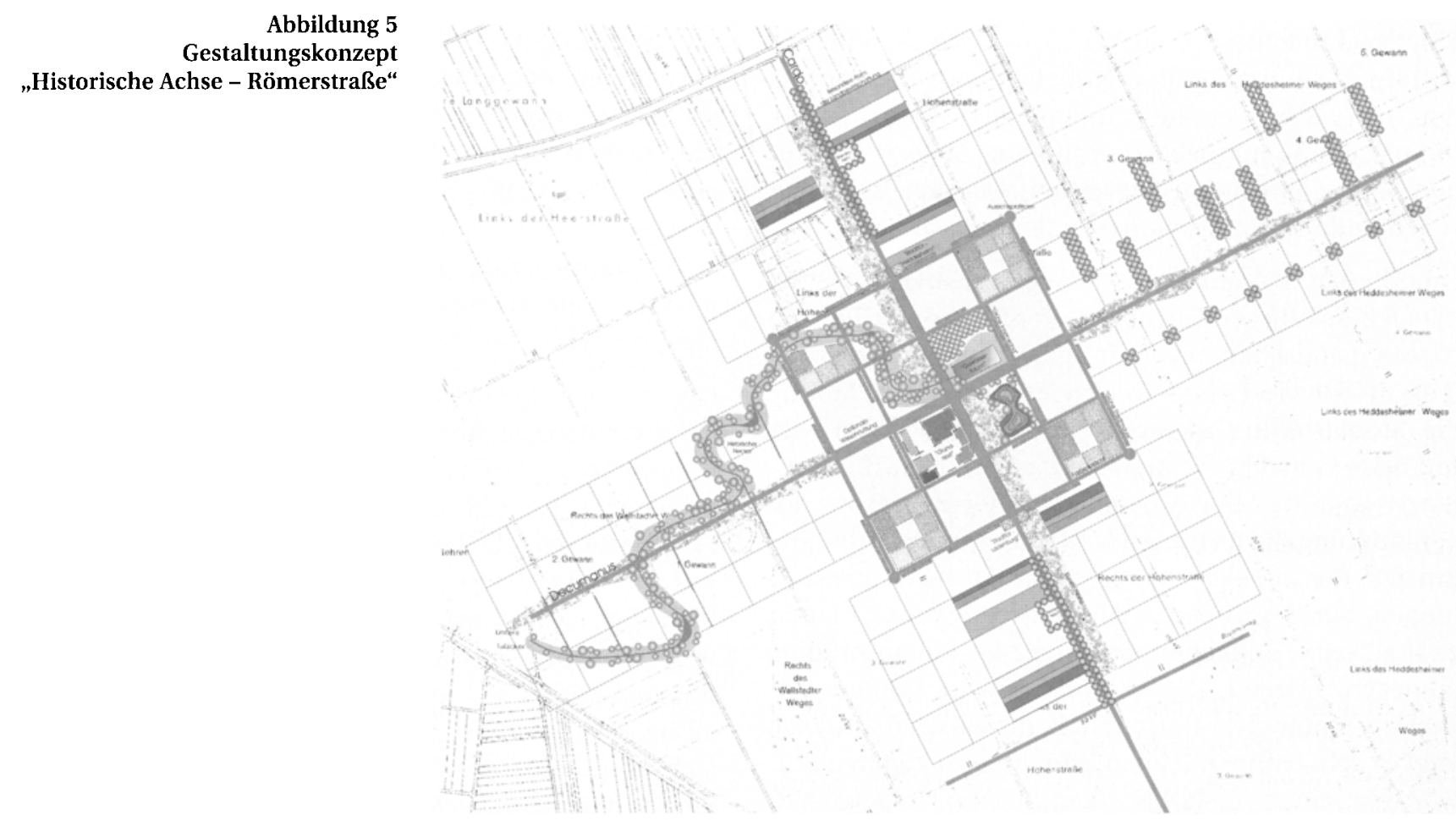

Entlang der Nord-Süd-Achse befindet sich ein so genannter „Erlebniskorridor“, der in regelmäßigen Abständen von Themenfeldern unterbrochen ist, welche das Thema "Römische Kultur in Natur und Landschaft" künstlerisch interpretieren. Das Umland ist nach römischem Vorbild in ein ländliches Gitternetz von $73 \times 73 \mathrm{~m}$ aufgeteilt. Es bildet die Basis für alle weiteren vorgenommenen Freiraumgestaltungen in diesem Bereich. So werden zum Beispiel die Baumreihen senkrecht zur „Decumanus-Achse“ in 73-Meter-Abständen angeordnet, womit die Perspektive des Wegeraumes betont wird.

Mit dieser Anlage soll ein stark anthropozentrisch geprägtes Gebilde entstehen, welches sowohl ökologischen, sozialen und mittelbar ökonomischen Ansprüchen (Landschaft als weicher Standortfaktor) Rechnung trägt. Zur Aufwertung dieses bisher kargen Landschaftsraumes wurden bestehende Zwänge (z.B. Grundbesitzverhältnisse) negiert, um visionäre Denkansätze zu ermöglichen. Diese Visionen könnten jedoch schon bald Realität werden (z.B. als externes Projekt der "Kleinen Landesgartenschau“ in Ladenburg).

\section{Schlussbetrachtung}

Die Übergangsphase in das 21. Jahrhundert war und ist geprägt von vielschichtigen Umwälzungsprozessen in allen Bereichen des täglichen Lebens. Globalisierung, Europäisierung sowie die fortschreitende Technisierung und der Wertewandel (Hübler 1988) erfordern auch in planerischer Hinsicht Antworten. In einem Europa der Regionen, in dem sich ein zunehmender Wettbewerb zwischen den Regionen und auch zwischen den Metropolen abzeichnet, wird es immer wichtiger, eine gewisse Unverwechselbarkeit, Tradition und Einzigartigkeit $\mathrm{zu}$ bewahren, die eigenen Schwächen zu erkennen sowie eigene Stärken auszubauen. Das sich allmählich herausbildende Regionalbewusstsein stellt die logische Reaktion zur voranschreitenden Globalisierung dar und leitet den ersten Schritt zu neuen Dimensionen in der Planung ein. Regionalisierung und Globalisierung stellen demnach neue Herausforderungen auch hinsichtlich der Sicherung und Entwicklung spezifischer Orts- und Landschaftsqualitäten dar.

Dieses Konglomerat an Veränderungen bleibt nicht ohne Konsequenz für die Landschaften Deutschlands. In Deutschland, als eines der am dichtesten besiedelten Länder Europas, ist Freiraum ein knappes und somit wertvolles Gut, das in besonderer Weise zu bewahren und zu weiterzuentwickeln ist. Zwei freiraumrelevante Entwicklungsrichtungen sind erkennbar: 
Einerseits macht sich ein Verfall der Landschaften in vorrangig ländlichen Räumen bemerkbar, andererseits dominiert in stark städtisch geprägten Bereichen die Intensivierung und Überformung der noch verbliebenen Landschaftsräume. Insgesamt gesehen, zeichnen sich deutliche Entmischungstendenzen ab.

Um diesem Negativtrend entgegenzuwirken, sollten die sich aus dieser Entwicklung ergebenden Chancen für die „Landschaften von morgen“ genutzt werden. In diesem Sinne ist es unerlässlich, künftig in der Planung die Mobilisierung endogener Kräfte zum Erhalt der Kulturlandschaften verstärkt voranzutreiben. Eine intensivere und aktivere Beteiligung der Bürger an der Veränderung ihres eigenen Umfeldes muss stattfinden, um z.B. der in Folge einseitiger struktureller Entwicklungen hervorgerufenen Abwanderung des „human capital“ aus ländlichen Regionen in Ballungsräume entgegenzuwirken. Die dadurch erzielte größere Akzeptanz unter den Betroffenen für Zukunftsprojekte vor Ort geht einher mit einer Zunahme an Eigenverantwortung, einer verstärkten Identifikationsbereitschaft eines jeden Einzelnen mit seinem Umfeld und der Verbesserung des Investitionsklimas.

Dieser stark kommunikative Ansatz ist insbesondere bei der Anwendung informeller Planungsinstrumente, wie z.B. eines Regionalen Landschaftsparks, von besonderer Bedeutung. Regionale Landschaftsparks ergänzen das oft starre und formale Instrumentarium und ermöglichen eine stärkere Projektorientierung. Diese von vielen Seiten eingeforderte Verschiebung -,weg von der Planung hin zu Projekten“- setzt Management voraus, um die Offenheit des Planungsprozesses kontrollieren und moderieren zu können. Besondere Erwähnung verdienen in diesem Zusammenhang Mediationsprozesse als Instrumente konfliktträchtiger Planungs- und Projektsteuerung, an deren Ende idealerweise eine so genannte "Win-Win-Situation“ steht, die allen Interessensgruppen, den Befürwortern und den Gegnern eines Projektes, Rechnung trägt. In diesem Fall besteht die Chance, mit dem informellen und inhaltlich flexiblen Instrument des Regionalen Landschaftsparks überzeugende Antworten auf die neuartigen Herausforderungen zu liefern. Der Regionale Landschaftspark dient sozusagen als Experimentierfeld für eine neue Planungs- und Verwaltungskultur ("Schlanke Planung", „Verwaltung 2000“ usw.).

In diesem Zusammenhang kommt der Kultur, als einen Baustein einer nachhaltigen Landschaftsentwicklung, eine große Bedeutung zu. Die kulturelle Ausgestaltung eines Landschaftsraumes wird im Wettbewerb der Regionen immer wichtiger. Kultur, hier Landschaftskultur bzw. Landschaftsästhetik, wird als weicher Standortfaktor immer stärker in wirtschaftliche Entscheidungs- prozesse einfließen: Sie kann, wenn entsprechende Investitionen getätigt werden, auch als mittelbare Wirtschaftsförderung angesehen werden. Hierbei gilt es, die Qualitäten einer Landschaft zu erkennen, zu sichern und auszubauen. An diesem Punkt knüpft die vorliegende Studienarbeit an und versucht dazu weiterführende Beiträge zu liefern. Sie stellt eine Diskussionsgrundlage für einen verstärkten planerischen Umgang mit dem Thema „Landschaftsästhetik“ dar.

Die vorliegende Arbeit sieht in der Idee des Regionalen Landschaftsparks Rhein-Neckar-Pfalz einen zukunftsweisenden Ansatz und eine Werkstatt für neue und zeitgemäße Handlungsansätze. In diesem Kontext ist es erforderlich, alle Ebenen im Sinne des Gegenstromprinzips miteinander logisch zu verknüpfen. Die inhaltlichen Zusammenhänge zwischen den Ebenen werden in dieser Studienarbeit herausgearbeitet und dokumentiert. Auf der Gesamtraumebene spielt der Vernetzungsgedanke die ausschlaggebende Rolle. Es soll dabei auf einen sinnvollen Zusammenhang der Strukturelemente rechts und links des Rheins innerhalb des Landschaftsparks geachtet und ein Handlungsrahmen gesetzt werden. Mit jedem tiefergehenden Arbeitsschritt wird der Umgang mit den Besonderheiten des Landschaftsraumes detaillierter. Das bedeutet: je größer der Maßstab, umso konkreter und intensiver die Auseinandersetzung mit den landschaftlichen Potenzialen.

Auf der Projektebene weist die Studienarbeit einen möglichen Weg zur Umsetzung kreativer Gestaltungskonzepte. Sie formuliert dabei eine visionäre „Landschaft von morgen“. Das Konzept stellt nicht nur das Ergebnis systematischer vorangegangener Arbeitsschritte dar, es ist zugleich Ausfluss subjektiven Empfindens und individueller Schöpfungskraft. Beim Konzept handelt es sich dabei jedoch nicht um etwas Absolutes oder Endgültiges, da es den Prototyp der "Landschaft von morgen" nicht gibt. Jeder Landschaftsraum erfordert eine individuelle, sensible und ganzheitlich nachhaltige Betrachtungsweise und definiert sich dem Betrachter/Bearbeiter immer wieder von neuem. Neben der Kreativität stellen Raum und Zeit unverzichtbare Komponenten im Planungsprozess dar, auf die von Fall zu Fall neu eingegangen werden muss. Pauschale Lösungen gibt es nicht.

Tragfähige Konzepte - ob formell oder informell brauchen sowohl den Blick zurück als auch den Blick in die Zukunft.

"Die Tragik in der modernen Landschaftsentwicklung ist nicht, dass das "Alte" verschwindet, wie es ja immer geschah, sondern das "Neue" der Landschaftskultur die falschen Wege weist. Zwar macht das Vergangene die Ge- 
genwart verständlicher, aber es ist eben kein Spiegel für den Weg in die Zukunft. Viele Fehler, die wir begehen oder in die wir geraten, geschehen, weil wir die Vergangenheit als Spiegel benutzen: wir schauen zurück, während wir uns vorwärts bewegen oder vorangetrieben werden. Die Landschaft der Zukunft bedarf neuer Phantasie!" (Haber 1998, S. 14)

\section{Literatur}

Bund; Misereor (Hrsg.) (1996): Zukunftsfähiges Deutschland, ein Beitrag zu einer global nachhaltigen Entwicklung. Studie des Wuppertal Instituts für Klima, Umwelt, Energie GmbH, Basel

Blöchliger, H. u.a. (1996): Schöne Landschaften: Was sind sie uns wert, was kostet ihre Erhaltung? Jahrbuch f. Ökologie 1996, S. $136-150$

Capra, F. (1999): Wendezeit, Bausteine für ein neues Weltbild, München

Ermer, K. (1996): Landschaftsplanung in der Stadt, Stuttgart

Fischer, K. (1997): Die unterschätzte Region - Der Rhein-NeckarRaum gestern und morgen, Mannheim

Grabow, B. u.a. (1995): Weiche Standortfaktoren, Stuttgart

Grub, H.; Lejeune, P. (1996): Grün zwischen Städten, München

Haber, W. (1998): Kulturlandschaft - Landschaftskultur. Welche Lehren lassen sich aus den Erfahrungen des 20. Jahrhunderts für den Umgang mit unserem Lebensraum ziehen? Vortrag anlässlich des 65. Geburtstages von Prof. Schmidt, Recklinghausen

Hübler, K.-H. (1997): Raumordnungspolitik und Wertewandel. Überlegungen zur Fortentwicklung der Raumordnungspolitik, Hrsg.: ARL - Akademie für Raumforschung und Landesplanung: Beiträge, Bd. 103, Hannover

Rothenburger, W. u.a. (1997): Bedeutung von Grünflächen in München (Ergebnisse einer Passantenbefragung), Lehrstuhl Wirtschaftslehre des Gartenbaues und Lehrgebiet Ökonomie der Landespflege, TU München

Schafranski, F. (1996): Landschaftsästhetik und räumliche Planung. = Materialien zur Raum- und Umweltplanung Nr. 85, Dissertation am Lehr- und Forschungsgebiet Landschafts- und Grünordnungsplanung, Universität Kaiserslautern, Kaiserslautern

Sieverts, T. (1998): Zwischenstadt - zwischen Ort und Welt, Raum und Zeit, Stadt und Land, Braunschweig / Wiesbaden

Stiens, G. u.a. (1999): Erhaltung gewachsener Kulturlandschaften als Grundsatz der Raumordnung. = Arbeitspapier des Bundesamtes für Bauwesen und Raumordnung, Bonn
Streich, B.; Kötter, T. (Hrsg.) (1998): Planung als Prozess - von klassischem Denken und Zukunftsentwürfen im Städtebau, Bonn

Tochtermann, D. u.a. (1997): IMMO Monitoring, Zürich

Wüst, H.-St.; Schafranski, F. (1999): Zukunftsinitiative „Unternehmen Grün“. Anstöße zu einer stärkeren Auseinandersetzung mit dem städtischen Grün als wirtschaftlicher Standortfaktor. Überarbeitete Fassung eines Vortrags im Rahmen des 1. Mannheimer Immobiliengespräches der Bayerischen Hypo- und Vereinsbank am 19.2.1999 in Deidesheim, Lehr- und Forschungsgebiet Landschafts- und Grünordnungsplanung, Universität Kaiserslautern

\section{Zeitschriftenartikel}

Curdes, G. (1989): Regionale Umstrukturierung durch weiche Standortfaktoren. Konzept zu einer regionalen Gestaltpolitik am Beispiel der Region Aachen. In: Raumforschung und Raumordnung, H. 4

Döhne, H.-J.; Walter, K. (1999): Aufgabe und Chance einer neuen Stadtentwicklungspolitik. Ziele und Konzeption des Bund-Länder-Programms „Stadtteile mit besonderem Entwicklungsbedarf - die soziale Stadt“. In: BBauBl, H. 5

Essing, H.; von Osten, W.-U. (1998): Naturschutz - wozu? In: Politische Ökologie, Mai/Juni

Hoisl, R. u.a. (1998): Naturbezogene Erholung als Motor der Landschaftsbildentwickung. In: Natur und Landschaft, Zeitschrift für Naturschutz, Landschaftspflege und Umweltschutz, H. 5

Rock, M. (1986): Ästhetischer Zugang zur Umwelt - Schönheit als Motiv des Naturschutzes. In: Natur und Landschaft, H. 12

Rock, M. (1983): Natur und Landschaft - was bedeuten sie für den Menschen? In: Natur und Landschaft, H. 7

Schafranski, F. (1999): Erläuterungen zur Stellungnahme des Arbeitskreises Landschaftskultur zum Entwurf des Umweltgesetzbuches (UGB-KomE), Kaiserslautern

Wolter, K. (1999): Kultur für die Region - Region für die Kultur. Thüringer Regionalplanertagung. In: ARL-Veranstaltungen, H. 2

Dipl.-Ing. Verena Mayer

Dipl.-Ing. Mark Schlick

Dipl.-Ing. Martin Groeger

Landschafts- und Grünordnungsplanung

Pfaffenbergstraße 95

67663 Kaiserslautern 